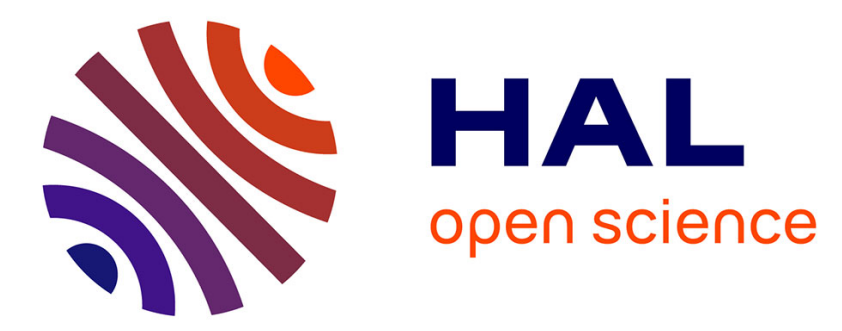

\title{
Exposure to pesticide residues from consumption of Italian blood oranges
}

Biagio Fallico, Mary Giuseppina d'Urso, Elena Chiappara

\section{To cite this version:}

Biagio Fallico, Mary Giuseppina d'Urso, Elena Chiappara. Exposure to pesticide residues from consumption of Italian blood oranges. Food Additives and Contaminants, 2009, 26 (07), pp.1024-1032. 10.1080/02652030902839731 . hal-00573875

\section{HAL Id: hal-00573875 \\ https://hal.science/hal-00573875}

Submitted on 5 Mar 2011

HAL is a multi-disciplinary open access archive for the deposit and dissemination of scientific research documents, whether they are published or not. The documents may come from teaching and research institutions in France or abroad, or from public or private research centers.
L'archive ouverte pluridisciplinaire HAL, est destinée au dépôt et à la diffusion de documents scientifiques de niveau recherche, publiés ou non, émanant des établissements d'enseignement et de recherche français ou étrangers, des laboratoires publics ou privés. 


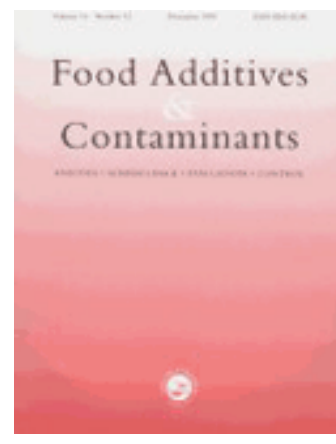

\section{Exposure to pesticide residues from consumption of Italian blood oranges}

\begin{tabular}{|r|l|}
\hline Journal: & Food Additives and Contaminants \\
\hline Manuscript ID: & TFAC-2008-330.R1 \\
\hline Manuscript Type: & Original Research Paper \\
\hline Date Submitted by the \\
Author: & 18 -Feb-2009 \\
\hline Complete List of Authors: & $\begin{array}{l}\text { Fallico, Biagio; Universita degli Studi di Catania, DOFATA } \\
\text { D'Urso, Mary } \\
\text { Chiappara, Elena; Università di Catania, DOFATA - sez. Tecnologie }\end{array}$ \\
\hline Methods/Techniques: & Chromatography - GC/MS \\
\hline Additives/Contaminants: & Pesticide residues \\
\hline Food Types: & Fruit, Vegetables \\
\hline \multicolumn{2}{|r}{} \\
\hline
\end{tabular}

\section{SCHOLARONE $^{\mathrm{m}}$ Manuscripts}




\section{Exposure to pesticides residues from consumption of Italian blood oranges} 2

3 B. Fallico*, M.G. D'Urso, E. Chiappara

4

5 DOFATA sez. Tecnologie Agro-alimentari, Università degli Studi di Catania, Italy 6

7 * Email: bfallico@ unict.it, Tel.0039957580214. 


\section{Abstract}

2 The paper reports the results of a 5-year study aimed to evaluate pesticide levels, deriving

3 from orchards activities, on the Italian most common orange cultivar (Citrus Sinensis, L.

4 Osbeck, cv. Tarocco). The study, using a Bayesian approach, allowed both the qualitative (the

5 number) and quantitative distributions (the amount) of pesticides to be determined with its

6 own probability value. Multi-residue analyses of 460 samples highlighted the presence of:

7 ethyl and methyl chlorpyrifos, dicofol, etofenprox, fenazaquin, fenitrotion, imazalil,

8 malathion and metalaxil-m. $30.5 \%$ of samples contained just one pesticide, $2.16 \%$ two

9 pesticides and $0.65 \%$ of samples had three pesticides at the same time. The most common 10 residue was ethyl chlorpyrifos followed by methyl chlorpyrifos.. The Estimated Daily Intake 11 (EDI) values for ethyl and methyl chlorpyrifos, as well as the distance from safety level 12 (NOAEL), were calculated. The risk has been differentiated in order: 1) to take account also 13 the period of real citrus consuming (180 days); 2) to differentiate the risk derived from eating 14 oranges that contain a certain level of chlorpyrifos from those unspecified. The most likely 15 EDI values for ethyl chlorpyrifos, derived from Italian blood orange consumption are: 0.01 16 and $0.006 \mathrm{mg} /$ day, calculated on 180 and 365 days, respectively. Considering the probability 17 of occurrence of ethyl chlorpyrifos, the EDI values are reduced up to: $2.6^{*} 10^{-3}$ and $1.3 * 10^{-3}$ $18 \mathrm{mg} /$ day, respectively. Analogously for methyl chlorpyrifos, the EDI most likely values are: 190.09 and $0.04 \mathrm{mg} /$ day, respectively. Considering its probability of occurrence the EDI values 20 are: $6.7 * 10^{-3}$ and $3.4 * 10^{-3} \mathrm{mg} /$ day, respectively. The results confirmed that levels of 21 pesticides in Italian Tarocco oranges deriving from a controlled chain are safe.

23 Keywords: EDI, NOAEL, Tarocco oranges, risk assessment, safety, @ risk, Monte Carlo. 


\section{Introduction}

2

3 The increase in recorded incidence of food-borne illness have driven the more industrialised

4 countries to develop more effective food safety control systems, based both on process 5 (design) and on performance-based (product) systems (Cho and Hooker, 2009). All food 6 operators are requested that they are managing food safety aspects correctly, developing 7 appropriate control procedures, analyses and monitoring activities. A risk-based approach is 8 strongly recommended. One of the most important aspect concerns the presence of 9 phytosanitary products.

11 The most comprehensive report of pesticide residues in Italy is prepared annually by an 12 important environmental association (Lega Ambiente, 2008). They reported the results of the 13 analysis of 10,048 vegetables and fruits carried out in 2007 by Italian Public Authorities 14 involved in food control (Chiappara and Fallico, 2008), and highlight that the number of 15 irregular samples of oranges declined from $1.3 \%$ to $1.0 \%$ but that $47.4 \%$ of samples contained 16 at least one pesticide. This improvement is significant also for Sicilian production, where of 1780 samples of analysed citrus (lemons and oranges): 57 (71.2\%) were pesticides free, 16 $18(20 \%)$ contained one pesticide, and 6 samples $(7.6 \%)$ had more than one pesticide residue. 19 The number of irregular samples in the citrus chain, according to results of national 20 monitoring plan for pesticides residues on food products (P.N.R.A.), co-ordinated by The 21 General Directorate for food safety and nutrition of Italian Ministry of Health, has decreased 22 significantly in the last decade. In fact, it declined from 5.6\% in 1993 to $1 \%$ in 2006.

24 The total Italian production of citrus fruit (medium value 2004-2006) is about 3.4 million 25 tons, with an invested area of 170.000 hectares. Sicily supplies $52.2 \%$ of orange production 
1 and $50 \%$ of Italian orange production comprises blood oranges; this percentage increases up

2 to $70 \%$ in Sicily where the cultivar Tarocco is predominant. (Tribulato, Maccarrone, La Rosa,

3 2007). In blood oranges there are many healthy substances, with antioxidant activity

4 (Maccarrone, 2004). In fact their juice contains anthocyanins, up to about $100 \mathrm{mg} / \mathrm{l}$ in freshly-

5 squeezed Tarocco juices (Arena et al. 2001), ascorbic acid up to about $800 \mathrm{mg} / \mathrm{l}$ (Maccarrone,

6 2004), hydroxycinnamic acids derivates like coumaric acid (Rapisarda et al., 1998).

7

8 The most widely used class of phytosanitary products in farming are the organophosphorus

9 compounds. They represent about the $40 \%$ or registered products. They can be very

10 dangerous as inhibit the normal action of acetylcholinesterase (AChE). The most common

11 one is chlorpyrifos [O,O-diethyl $O$-(3,5,6-trichloro-2-pyridinyl) phosphorothionate].

12 Alternatively in post-harvest treatment thiabendazole and imazalil are currently applied to

13 control mould decay in citrus fruit (Schirra et al., 1996).

14

15 Many studies have been carried out in order to evaluate the decay kinetics of some pesticides 16 currently used on citrus fruit. Montemurro et al. (2002) found that degradation kinetics of 17 chlorpyrifos depends on commercial formulation as well as for fenitrothion (Montemurro et 18 al., 2005). Schirra et al. (2005) evaluated the residue levels, as well as persistence and storage 19 performance of citrus fruits treated with fludioxonil.

21 The importance of a risk-based approach to guarantee food safety/quality is strongly 22 recommended both by Regulatory Agencies (Reg. CE 852/04; FAO, 2006) and other bodies 23 (ISO 22000:05; GLOBAL/GAP). Many methods have been proposed to perform risk 24 assessment. The simplest ones, qualitative or semi-quantitative, take in account just the 25 probability and the consequences (impact) of the negative event(s). Exhaustive methods to 
1 perform risk assessment, from general applications to food safety/quality, are available in

2 literature (Tennant, 1997; Vose, 2000; FAO, 2006). Risk assessment is based both on

3 deterministic approach when the calculated risk is based on a point estimate, usually the

4 worst-case value for each input variable; instead in a probabilistic risk assessment model both

5 variability and uncertainty in input variables can be taken into consideration, by using

6 probability distributions to represent the input variables instead of point estimates (Vose,

7 2000; Spanjersberg et al. 2007). Vose (1998) proposed a QRA, based on Monte Carlo

8 simulation method, to evaluate microbial food safety. The probabilistic approach has been

9 used by Slob and Pieters (1998) to estimate human acceptable exposure limits (RfD, ADI,

10 TDI) using distributions for extrapolation factors instead of single uncertainty factors, more 11 conservative; beside Slob (2006) published a study on the probabilistic dietary exposure 12 assessment taking into account variability in both amount and frequency of consumption. The 13 probabilistic model has been also used (Spanjesberg et al., 2007) for allergen risk assessment, 14 resulting in a more exhaustive risk assessment respect to traditional deterministic risk 15 assessment. Beside probabilistic modelling has been used for exposure assessment of operator 16 and residential non-dietary exposure for pestidide products (Lunchick, 2001), for cumulative 17 risk assessment of the exposure to organophosphorus and carbamate insecticides in the Ducht 18 diet (Boon et al., 2008) and also to estimate children's short-term residential exposure to lead 19 (Canales, Leckie, 2007).

21 The aims of this paper were: a multi-annual evaluation, both qualitative and quantitative, of 22 pesticide residues on blood oranges (Citrus Sinensis L. Osbeck, Cl Tarocco); to estimate the 23 daily intake of residues by consumption of blood oranges; the probability of consumers' 24 overexposing to exposure levels above the TDI. 


\section{$1 \quad$ Materials and methods}

2 This study has been carried out in collaboration with the Italian leading company of blood 3 oranges (Oranfrizer, Scordia $(C T)$ ). It produces both for the internal market and, overall, for

4 the international one (UK, Germany, Japan, etc.). The company in the last decade has adopted 5 many quality schemes, as well as: farm inspections and chemical controls of product.

Sampling and Multiresidual analysis

8 During the 5-years of the study (2003-2007), 460 samples were collected as indicated in

9 Table 1. Within 48 hours from picking, in triple, a multi-residue analysis was carried out. 10 According to ISTISAN 97/23 protocol. A purée was obtained from $1 \mathrm{~kg}$ of oranges. Puree (10 $11 \mathrm{~g})$ was mixed with diatomaceous earth (13 g), transferred into a glass column $(20 \times 200 \mathrm{~mm})$ 12 and eluted with four aliquots (25 ml each) of a mixture dichloromethane-ethylacetate (50-50). 13 The eluted sample was dried under reduced pressure, re-dissolved with methanol (1 ml) and 14 an aliquot $(0.1 \mu \mathrm{L})$ injected to GC-MS in splitless conditions.

16 The GC-MS system was a SHIMADZU GC 17A-MD 5800, equipped with a column SLB $^{\mathrm{TM}}$ $175 \mathrm{~ms}\left(\mathrm{~L} \times\right.$ I.D. $\left.30 \mathrm{~m} \times 0.25 \mathrm{~mm}, \mathrm{~d}_{\mathrm{f}} 0.25 \mu \mathrm{m}\right)$, with a linear flow of $1.0 \mathrm{ml} / \mathrm{min}$, a linear 18 velocity of $36.3 \mathrm{~cm} / \mathrm{sec}$. The sampling time was of $5.0 \mathrm{~min}$., the injector temperature was of $19270.0{ }^{\circ} \mathrm{C}$. The oven temperature program was initially set at $50.0^{\circ} \mathrm{C}$ with a hold of $1 \mathrm{~min}$, 20 after was ramped to $120.0^{\circ} \mathrm{C}$ at $25^{\circ} \mathrm{C} / \mathrm{min}$, with a hold of $1 \mathrm{~min}$ and finally was ramped to $21300.0{ }^{\circ} \mathrm{C}$ at $5{ }^{\circ} \mathrm{C} / \mathrm{min}$, with a hold of $13.20 \mathrm{~min}$. The total oven program was of $53.0 \mathrm{~min}$. The 22 interface temperature of detector was of $280.0^{\circ} \mathrm{C}$ and the range mass was from $54.00 \mathrm{~m} / \mathrm{z}$ to $23580.0 \mathrm{~m} / \mathrm{z}$, working in Selected Ion Monitoring (SIM) target qualifier ions were used, both for 24 the identification and quantification of each compound. Recovery (\%) and reproducibility $25\left((\mathrm{RSD})_{\mathrm{R}} \%\right)$, for each of the detected compounds, were studied at four fortification levels 
$1 \quad(0.001-1.00 \mathrm{mg} / \mathrm{kg})$, for a total of 8 replicates. All but one, fenazaquin $(80 \%)$, the recovery

2 was always higher than $90 \%$. RSD values are reported in table 3.

3

4 Statistics and risk analysis were carried out with the software Excel 2003 (Microsoft Inc.) and $5 @$ Risk (ver. 4.5, Palisade Inc.), respectively. All distribution are analysed between the $5^{\circ}$ and $695^{\circ}$ percentiles.

7

8 Qualitative Analysis

9 For each of the studied years and for the total of 5-years, the number and kind of 10 phytosanitary products on each orange sample was evaluated. To this purpose, all analytical 11 results where reported in an excel spreadsheet, assigning: the value one when the chemical 12 compound was present, zero in all others cells. According to Vose (1998), data on 13 presence/absence (successes or failures) have been used as inputs to determine the probability 14 of an event occurs. The Beta distribution describes the true probability (p) of an event 15 occurring, given (n) and (s) obtained from spread sheets, respectively (Vose, 1998). In order 16 to estimate the number of phytochemicals present at the same time on Tarocco oranges (up to 17 6), and each associated probability, a Binomial distribution was used. The distributions are : $18 \mathrm{p}=\operatorname{BETA}(\mathrm{n}+1 ; \mathrm{n}-\mathrm{s}+1) ; \mathrm{s}=\operatorname{Binomial}(\mathrm{n} ; \mathrm{p})$

19 This qualitative analysis was carried out in order to evaluate the probability of finding: ethyl 20 chlorpyrifos, methyl chlorpyrifos, or both of them.

\section{Quantitative Analysis}

22 The aim of this part of the study was, for each and the five years, to evaluate the level $23(\mathrm{mg} / \mathrm{kg})$ of each phytochemical present in Tarocco oranges. Moreover for each phytochemical 24 the associated best-fitted distribution was obtained. For each sample and for each 25 phytochemical in Table 3 are reported: the RSD, the number and the percentage of positive 
1 samples, the MRL, the average value, the standard deviation, the minimum and maximum

2 value, the best fitted distribution.

3 Risk Assessment

4 For the most important residues found in blood oranges the Estimated Daily Intake (EDI) and 5 the Potential risk (EDI /NOAEL) for consumers were calculated for the period 2003-2007. In 6 both cases a probabilistic approach was used (@Risk, ver. 4.5, Palisade Inc.). The EDI (Food 7 chemical concentration $\mathrm{x}$ food consumption) was estimated according to Douglass and 8 Tennant (1997) multiplying the distribution of the phytochemical concentration $(\mathrm{mg} / \mathrm{kg})$ in

9 Tarocco oranges with the distribution of orange pro capita consumption in Italy (ISMEA, 10 2008) in the same period. The simulations were run, as single, for ethyl chlorpyrifos and 11 methyl chlorpyrifos, as multiple intakes for the sum of ethyl and methyl chlorpyrifos.

12

13 In order to characterise the risk (FAO, 2006), the NOAEL (Non Observed Adverse Effect 14 Level), the highest tested dose on animals without adverse effect, was obtained from literature 15 (INCHEM, 1999). Analogously to other food risk assessment studies (Spanjersberg et al., 16 2007; Zhang et al., 2008), the potential risk for consumers, the relationship between the 17 chemical intake distribution and the safety factors distribution for both ethyl and methyl 18 chlorpyrifos as single, and as multiple intakes for the sum of them, was estimated dividing the 19 two distributions (in practice how far are the pesticides concentrations on oranges from 20 safety concentrations).

22 In order to use the same units of EDI, mg/day, safety factor NOAEL was multiplied for $60 \mathrm{Kg}$ 23 (the average weight of a person) (WHO and FAO, 2004).

24 In all risk analyses the following assumptions were done: 
1. Pesticides analyses on oranges, according to official methods (ISTISAN, 97/23), must be carried out on the entire fruit (peel and edible portion). In Tarocco oranges the ratio between flavedo + albedo $=$ peel and edible portions (pulp) is around: 10/90; while the most part of pesticides remains on flavedo, only a small amount, usually $3-5 \%$, transfers into the albedo (edible portion). As the strong difference in pesticides concentrations between the peel and the edible portion is largely compensated by the ratio between the two parts: the data concerning pesticides have not been modified.

2. Usually the consumption pro capite of a food commodity is calculated per year (365 days), as the fresh oranges consumption is concentrated between December and May, a 6 months (180 day) pro capite consumption, and relatives simulations, have been also arbitrary calculated.

3. Lastly, in order to differentiate the risks by pesticides exposure of the generic population by the consumer that really eat Italian Tarocco oranges, one more input has been added. In fact, the distribution of pesticide(s) concentration has been multiplied for the distribution of probability of the presence of the pesticide(s) (Output of qualitative analysis). 


\section{$1 \quad$ Results and discussion}

2

3 Multi-residue analysis of 460 samples of Italian Tarocco oranges, for the years 2003-2007,

4 highlighted the presence of the following pesticides: ethyl and methyl chlorpyrifos, dicofol,

5 etofenprox, fenazaquin, fenitrotion, imazalil, malathion, metalaxil-m.

\section{$6 \quad$ Qualitative Analysis}

7 Orange samples, for each year, were analysed in order to know the probability to have, at the

8 same time, one, two or three pesticides residues. Results of the binomial distribution, are 9 reported in Table 2.

10 Of the analysed samples, over the five studied years, $140(30.5 \%)$ contained just one 11 pesticide; 9 samples two pesticides $(2.16 \%)$; two samples 3 pesticides $(0.65 \%)$. None of the 12 samples had four, or a higher number, of pesticide residues. Moreover, the probability 13 distributions (Figure 1a) highlighted that: although 140 is the most probable number, it must 14 be expected that Tarocco oranges with just one pesticide will fall in the range between: 12415157 (26.95-34.13\%); and between 5 and 15 (1.08-3.26\%) samples with two pesticides (Figure $161 \mathrm{~b})$, and 1 to $6(0.22-1.30 \%)$ with three (Figure 1c). In 2003, 11 samples $(16.7 \%)$ contained 17 just one pesticide, with an acceptable range between 7-17 (Table 2). In this case the most 18 common pesticide was ethyl chlorpyrifos, followed by methyl chlorpyrifos and three samples 19 containing: dicofol, etofenprox and malathion, respectively. The two samples with two and 20 three pesticides contained ethyl and methyl chlorpyrifos; ethyl, methyl chlorpyrifos plus 21 fenanzaquin, respectively.

23 Of the 92 samples analysed in 2004, 31 contained one pesticide: ethyl chlorpyrifos. Just one 24 sample contained two pesticides: ethyl chlorpyrifos and dicofol at the same time. The 
1 probability distribution, concerning the presence of one pesticide, has shown acceptable range

2 between 24 and 39 samples; and up to 4 samples for the presence of two pesticides (Table 2).

3 In 2005, 43.7\% of samples contained just one pesticide, distributed as follows: 47 samples

4 ethyl chlorpyrifos, 19 methyl chlorpyrifos, 2 metalaxil-m, 1 with fenitrotion. The $3.75 \%$

5 showed at the same time two pesticides, distributed as follows: 2 samples with ethyl and 6 methyl chlorpyrifos, one sample each: methyl chlorpyrifos and metalaxil-m; ethyl 7 chlorpyrifos and etofenprox; etofenprox and fenazaquin.

9 In 2006 the $18.6 \%$ of samples contained just one pesticide, distributed as follows: 14 samples 10 ethyl chlorpyrifos, two samples fenitrotion, one with methyl chlorpyrifos and etofenprox, 11 respectively. Two samples contained ethyl and methyl chlorpyrifos. One sample at the same 12 time contained methyl chlorpyrifos, dicofol and fenitrotion.

In 2007 the $28.6 \%$ of samples contained one pesticide residue of which 6 samples contained 15 ethyl chlorpyrifos and 5 samples contained imazalil.

17 All pesticides found in Tarocco oranges, in the five years which were studied conformed with the legislation (Ministerial Decree of 27/08/2004 ).

As chlorpyrifos, both as ethyl and methyl, was the most commonly occurring residue, a 21 specific qualitative analysis was carried out in order to evaluate the probability as single or in 22 co-presence. Results are in figures $2 \mathrm{a}, 2 \mathrm{~b}$ and $2 \mathrm{c}$.

24 In particular, the $23.16 \%$ of samples have shown only ethyl chlorpyrifos residues, with a 25 probability of about $4.5 \%$, with an acceptable range between $20 \%$ and $26.5 \%$ (figure $2 \mathrm{a}$ ); 
1 instead the $7.8 \%$ of samples have shown only methyl chlorpyrifos residues, with a probability

2 of about $7 \%$; this probability distribution, reported in figure $2 \mathrm{~b}$, has shown acceptable ranges

3 between 27 and 46 samples, corresponding to $5.87 \%$ and $10 \%$ respectively. The most

4 probable number of samples with ethyl and methyl chlorpyrifos, in the five studied years, is

5 equal to 6 , representing the $1.5 \%$ of samples (figure $2 \mathrm{c}$ ); the acceptable range is between

$6 \quad 0.65 \%$ and $2.6 \%$.

7

\section{$8 \quad$ Quantitative Analysis}

9 Table 3 reports the results of quantitative analyses, concerning: ethyl and methyl chlorpyrifos,

10 dicofol, etofenprox, fenazaquin, fenitrotion, imazalil, malathion and metalaxil-m, calculated

11 in positive orange samples. Moreover the table reports, for each molecule, the admitted

12 Maximum Residue Level in oranges and, when the pesticide was present at least in five 13 samples, the best fitted distribution.

As described above, ethyl and methyl chlorpyrifos are the most commonly coccuring 16 pesticides, deriving from farming activities, in blood oranges followed by etofenprox and 17 fenithrotion. The presence of imazalil (Table 3) must be discussed separately. In fact, it is 18 present in a very low number of samples compared to literature data (Fernandez et al., 2001). 19 This is normal if we consider that it is not used during farming. But, is the most used post20 harvest fungicide in citrus fruit, given with the waxing of product. The presence of this 21 molecule at this stage of the chain may indicate that a few lots are not supplied directly by 22 farmers but from other citrus business operators.

24 The average level of ethyl chlorpyrifos was: $0.07 \mathrm{mg} / \mathrm{kg}$ of oranges, with the lowest value, in 25 positive samples, of $0.003 \mathrm{mg} / \mathrm{kg}$ and the highest value of $0.29 \mathrm{mg} / \mathrm{kg}$. The concentration was 
1 always lower than the MRL and was normal distributed. The average level of methyl 2 chlorpyrifos was $0.56 \mathrm{mg} / \mathrm{kg}$, with the highest value, measured in 2005 , of $2.05 \mathrm{mg} / \mathrm{kg}$. The 3 last value was four fold higher the MRL (Table 3). Also methyl chlorpyrifos was normally

4 distributed. Dicofol was present in three samples over the five studied years with an average 5 value of $6.69 \mathrm{mg} / \mathrm{kg}$; but, in 2003, one sample showed, with $20 \mathrm{mg} / \mathrm{kg}$, the highest measured 6 concentration of pesticide; this value was ten fold higher than the MRL value; instead, in 7 2004, one sample showed, with $0.07 \mathrm{mg} / \mathrm{kg}$, a concentration twenty-eight times lower than 8 MRL, and in 2006 seventy-four times lower. All but one of the remaining pesticides have

9 shown very low concentrations and far from MRL. The only exception was imazalil with an 10 average value of $3.3 \mathrm{mg} / \mathrm{kg}$ and the highest measured value, $4.8 \mathrm{mg} / \mathrm{kg}$, next to the MRL 11 (Table 3).

12

13 Data shown above are qualitatively similar to pesticides residues found in Spanish oranges 14 (Fernandez et al., 2001), but quantitatively shown some differences. In fact, without 15 considering differences of imazalil, explained above, it is possible to see that: ethyl 16 chlorpyrifos is largely used in Italian samples than in Spanish ones, but at lower levels, 0.07 $17 \mathrm{mg} / \mathrm{kg}$ and $0.16 \mathrm{mg} / \mathrm{kg}$, respectively; Dicofol was present in $18.7 \%$ of the Spanish with an 18 average value of $0.28 \mathrm{mg} / \mathrm{kg}$, while in Italian Tarocco oranges was present in the $0.65 \%$ of 19 samples with an average value, excluding the sample with $20 \mathrm{mg} / \mathrm{kg}$, of: $0.04 \mathrm{mg} / \mathrm{kg}$. 20 Fernandez et al. (2001) report a 4\% of samples above the MRL. The results of the present 21 investigation report the $0.44 \%$ of samples exciding the MRL values. Even lower the 22 percentages reported for 2006 and 2007 of: 1\% and 1.3\%, respectively (PNRA, 2006; Lega 23 Ambiente, 2008). But, two considerations must be done: first, the present investigation is 24 addressed to evaluate risk coming from farming activity, risk from post harvesting (e.g., 
1 imazalil) are deliberately neglected; afterwards, data concerning this investigation refer to a

2 controlled citrus chain, for instance, street food is not taken in account.

3

4 Assessment of EDI and EDI /NOAEL ratio.

5 The first two simulations were aimed to:

6 respectively;

- estimate the most probable EDI values, and their distributions, for ethyl chlorpyrifos, for methyl chlorpyrifos and the sum of two pesticides from consuming Italian Tarocco oranges, considering a consumption distributed on 365 and 180 days,

- estimate the EDI values as described above, but keeping in account also the probability distribution of the presence of the pesticide (qualitative analysis).

In practice, the first one describe the EDI of a consumer that is going to eat an orange having a certain level of chlorpyrifos, the second one the EDI of consumer that is going to eat an orange, without knowing if it contains or not pesticides.

When a Tarocco orange contain a certain level of ethyl chlorpyrifos, the most probable EDI values, calculated on 180 or 365 days of consumption, are: $0.01 \mathrm{mg} / \mathrm{day}$ or $0.006 \mathrm{mg} / \mathrm{day}$, respectively. Moreover the EDI distributions say that, acceptable values can range from 0 up to $0.03 \mathrm{mg} / \mathrm{day}$, for 180 days, the half for 365 days. EDI of ethyl chlorpyrifos values have a substantial decrease if we consider that this pesticide is not always present in oranges. In fact, EDI values are: $2.6^{*} 10^{-3}$ and $1.3 * 10^{-3} \mathrm{mg} /$ day, calculated at 180 and 365 days, respectively. The WHO/FAO report on pesticides residues in food (2004), assign a daily intake in Europe, of ethyl chlorpyrifos from citrus, of $3.6^{*} 10^{-3} \mathrm{mg} /$ day. This value is significantly higher, 2.8 fold, than the analogous reported above $\left(1.3 * 10^{-3} \mathrm{mg} / \mathrm{day}\right)$. The difference could be ascribed 
1 to the lower level found in blood oranges (Table 3) compared to other citrus (Fernandez et al., 2 2001).

4 For methyl chlorpyrifos residues found in our samples, the four simulations indicate that the most probable EDI values (on 180 or 365 days of consumption) are of $0.09 \mathrm{mg} /$ day or 0.04 mg/day, respectively. Furthermore acceptable values of EDI distributions can range from 0 up to $0.23 \mathrm{mg} / \mathrm{day}$, for 180 days, from 0 up to 0.12 for 365 days. If we consider that methyl chlorpyrifos is not always present in oranges, EDI values have a considerable reduction; in fact they are: $6.7 * 10^{-3}$ and $3.4 * 10^{-3} \mathrm{mg} /$ day, calculated at 180 and 365 days, respectively.

Data shown above highlighted that the daily intake of methyl chlorpyrifos, from blood 11 oranges, is always higher than ethyl chlorpyrifos, up to 7.6 fold.

13 As methyl chlorpyrifos has been found in some samples, also the presence of, a multiple 14 intake (sum of ethyl and methyl chlorpyrifos), was estimated. In both cases: keeping in 15 account the distribution of the averages levels and the probability distribution that the two 16 molecules are contemporarily in the same sample. Considering just the concentrations 17 encountered in our samples the estimated daily intake increases significantly up to: 0.03 and $180.01 \mathrm{mg} /$ day for 180 and 365 day, respectively. But, keeping in account the probability 19 distribution that this event occurs, the co-presence in the same sample, the EDI values go 20 down up to: $4 * 10^{-4}$ and $2 * 10^{-4} \mathrm{mg} /$ day, respectively.

22 The ADI (Acceptable Daily Intake) value for chlorpyrifos is in the range $0-0.01 \mathrm{mg} / \mathrm{kg} \mathrm{bw}$ 23 (INCHEM, 1999). For a person of $60 \mathrm{~kg}$ of body weight, the highest acceptable daily intake 24 is: $0.6 \mathrm{mg}$. This means that, in the worst case, using EDI at 180 days, the consumption of 
1 Italian Tarocco oranges can contribute to the $1.87 \%, 14.37 \%$ and $4.71 \%$, of ADI as ethyl

2 chlorpyrifos, methyl chlorpyrifos and the sum of ethyl and methyl, respectively.

3

4 Results of simulations aimed to verify the distance, in terms of distributions probability, 5 among the EDI values and the NOAEL values, indicate that, even in the worst case, for the 6 presence of ethyl chlorpyrifos, considering a consumption of oranges during 6 months (180 7 days), the ratio (EDI/NOAEL) has a $0.48 \%$ as average value and a highest acceptable value 8 of $1.7 \%$. Considering the probability that chlorpyrifos may be not present, the ratio is 0.1 or $90.05 \%$ for 180 or 365 days, respectively.

11 For the presence of methyl chlorpyrifos, the ratio (EDI/NOAEL 180 days) has a $3.7 \%$ as 12 average value and a highest acceptable value of $14 \%$, if we considering the probability that 13 methyl chlorpyrifos may be not present, the ratio decrease significantly to $0.29 \%$ or $0.14 \%$ 14 for 180 or 365 days, respectively. The cumulative analysis shows that the ratio (EDI/NOAEL 15180 days) has a $1.19 \%$ as average value and a highest acceptable value of $4.9 \%$; besides if we 16 considering the probability that methyl and ethyl chlorpyrifos may be not present 17 contemporarily in the same sample, the ratio decrease significantly to $0.02 \%$ or $0.01 \%$ for 18180 or 365 days, respectively.

20 The above simulations have shown that chlorpyrifos levels are always largely in the safety 21 area.

\section{Conclusions}

24 This study highlighted the following findings: 
- The number, the associated probability and distribution of pesticides in Tarocco

2 oranges;

- The most common pesticide residue for farming activity is ethyl chlorpyrifos, followed by methyl chlorpyrifos;

- All but two of the 460 analysed samples shown pesticide levels below the MRL;

- The Estimated Daily Intake of methyl and ethyl chlorpyrifos, and the sum of two, from eating Tarocco Blood oranges has been estimated;

- Authors believe the EDI, calculated considering a citrus eating spread of 180 days, more realistic than that calculated on 365 days;

- The percentage of ethyl chlorpyrifos, for a person of $60 \mathrm{~kg}$ of body weight, in comparison ADI has been calculated;

- Both for ethyl and methyl chlorpyrifos, as well as for their sum, the measured levels in oranges, and, as consequence, the estimated consumer intake is far from NOAEL value.

Moreover, data reported above, in our opinion, have shown the efficacy of performance-based 16 (product) control systems (Cho and Hooker, 2009) and of the co-regulation approach 17 (Martinez et al., 2007) to guarantee food safety. The acceptable level of pesticides could be 18 ascribed also to the pressure of Public Authorities on business operators and, as a 19 consequence, the practical capacity of citrus chain actors to control it. In fact, also when 2 of 20 the 460 samples where outlaw they have been intercepted not representing a real risk for 21 consumers. Studies concerning post-harvesting citrus activities, as well as, the not controlled 22 (farmers' direct selling, street or small shops) are in progress. 


\section{References}

2 Arena E, Fallico B, Maccarrone E. 2001. Evaluation of antioxidant capacity of blood orange

3

4

5

6

7

8 juices as influenced by constituents, concentration process and storage. Food Chemistry 74: 423-427.

Boon Pe, Van der Voet H, Van Raaij Mtm, Van Klaveren Jd. 2008. Cumulative risk assessment of the exposure to organophosphorus and carbamate insecticides in the Ducht diet. Food and chemical toxicology 46: 3090-3098.

Canales R, Leckie JO. 2007. Application of a stochastic model to estimate children's shortterm residential exposure to lead. Stoch. Environ. Res. Risk Assess. 21: 737-745.

Chiappara E, Fallico B. 2008. Analysis of the Italian Public Food Control System and its relationship to European hygiene and safety legislation. Italian Journal of Food Science vol. 20: 297-320.

Cho B, Hooker N. 2009. Comparing food safety standards. Food Control 20: 40-47.

Douglass J.S., Tennant D.R.1997. Estimation of dietary intake of food chemicals. In Chapman \& Hall (Ed.), Food Chemical Risk Analysis (pp.212-215), chap. 8.

FAO and WHO. 2006. Food safety risk analysis. A guide for national food safety authorities. FAO FOOD AND NUTRITION PAPER 87.

INCHEM 1999 available from: http://www.inchem.org/

ISTISAN 97/23. National Health Institute. 1997. Multiresidue analytical procedures for pesticides residues in vegetable products. Sections: A (pp. 3-5, C.2.3. (pp 32-33). http://www.iss.it/publ/rapp/cont.php?id=656\&lang=1\&tipo=5\&anno=1997

Fernández M, Picó Y, Mañes J. 2001. Pesticides residues in oranges from Valencia (Spain). Food Additives and Contaminants 18: 615-624. 
1 Italian Ministry of Health. Ministerial Decree 27 August 2004. Phytochemicals: Maximum

2 residue levels of active substances on food products. Italian Republic Official Gazette No 292, 14/12/2004,O.S. No. 179.

Italian Ministry of Health. 2007. Official Control of the Phytosanitary Products Residues in Fruit and Vegetables. Results for 2006. Available from: http://www.ministerosalute.it/alimenti/resources/documenti/sicurezza/anno_2006.pdf.

ISMEA. April 2008. Citrus Consumption in Italy. Available from: http://www.ismea.it/flex/cm/pages/ServeBLOB.php/L/IT/IDPagina/3051

Lega Ambiente: Dossier Pesticidi nel Piatto 2008. 23 Maggio 2008. Available from: http:// www.legambiente.eu/documenti/2008/0526_pesticidi_nel_piatto_2008/0526_Pesticidi2 008.pdf

Lunchick C. 2001. Probabilistic Exposure Assessment of operator and residential non-dietary exposure. Ann. Occup. Hyg., 45: S29-S42.

Maccarrone E. 2004. Composition and healthy value of Sicilian blood oranges juice. Rivista di frutticoltura e di ortofloricoltura. Volume LXVI - N.3 - Marzo 2004 (25:27).

Martinez M.G, Fearne A, Caswell J.A, Henson S. 2007. Co-regulation as a possible model for food safety governance: Opportunities for public-private partnerships. Food Policy 32: 299-314.

Montemurro N, Grieco F, Lacertosa G, Visconti A. 2005. Persistence of Fenitrothion in oranges and clementines after treatment with emulsifiable concentrate and microencapsulate formulations. Food Additives and Contaminants 22: 39-47.

Rapisarda P, Carollo G, Fallico B, Tomaselli F, Maccarrone E. 1998. Hydroxycinnamic acids as markers of Italian blood orange juices. Journal of Agricolture and Food Chemistry 46: 464-470. 
1 Schirra M, Cabras P, Angioni A, Melis M. 1996. Residue level of Imazalil fungicide in 2 lemons following prestorage dip treatment at 20 and $50{ }^{\circ} \mathrm{C}$. Journal of Agricultural and $3 \quad$ Food Chemistry 44: 2865-2869.

4 Schirra M, D’Aquino S, Palma A, Marceddu S, Angioni A, Cabras P, Scherm B, Migheli Q. 5 2005. Residue level, persistence and storage performance of citrus fruit treated with fludioxonil. Journal of Agricultural and Food Chemistry 53: 6718-6724.

Slob W. 2006. Probabilistic dietary exposure assessment taking into account variability in both amount and frequency of consumption. Food and Chemical Toxicology 44: 933951.

Slob W, Pieters M.N. 1998. A probabilistic approach for deriving acceptable human intake limits and human health risks from toxicological studies: general framework. Risk Analysis 18: 787-798.

Spanjersberg M.Q.I, Kruizinga A.G, Rennen M.A.J, Houben G.F. 2007. Risk assessment and food allergy: the probabilistic model applied to allergens. Food and Chemical Toxicology 45: 49-54.

Tennant D.R. 1997. Food Chemical Risk analysis. First Edition. England. Chapman \& Hall (Ed.)

Tribulato E, Maccarrone E, La Rosa G. 2007. Italian citrus cultivation. The present time and prospectives. In Gruppo Perdisa Edition, New frontiers of Italian arboriculture (pp. 205221).

Vose D.J. 1998. The Application of quantitative risk assessment to microbial food safety. Journal of Food Protection 61: 640-648.

Vose D.J. 2000. Risk Analysis. A quantitative guide. $2^{\text {nd }}$ ed. England. John Wiley \& Sons, Ltd.

WHO and FAO. Pesticides residues in food-2004. Report 2004. FAO, Plant Production and 
1 protection paper 178. Rome, Italy, 20-29 September 2004. Available from:

2 http://www.fao.org/ag/agp/agpp/Pesticid/p.htm

3 Zhang XF, Driver JH, Li YH, Ross JH, Krieger RI. 2008. Dialkil phosphates (DAPs) in fruits and vegetables may confound biomonitoring in organophosphorus insecticide exposure and risk assessment. Journal of Agricultural and Food Chemistry 56: 10638-10645.

6

7

8

9 FIGURE CAPTIONS

10 Figure 1:

11 a. Probability distribution of presence - absence , 1 pesticide residue 2003-2007

12 b. Probability distribution of presence-absence, 2 pesticide residues 2003-2007

13 c. Probability distribution of presence-absence, 3 pesticide residues 2003-2007

14

15 Figure 2:

16 a. Probability distribution of presence-absence, ethyl chlorpyrifos 2003-2007

17 b. Probability distribution of presence-absence, methyl chlorpyrifos 2003-2007

18 c. Probability distribution of presence-absence, ethyl + methyl chlorpyrifos 2003-2007 


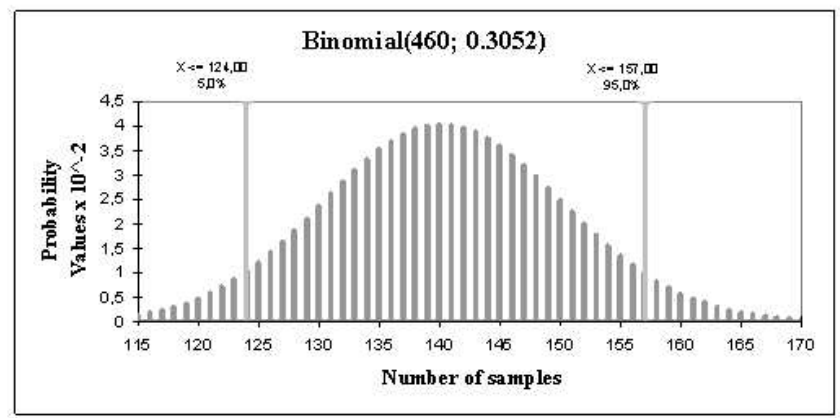

Figure 1a. Probability Distribution of presence-absence, 1 pesticide residue 2003-2007

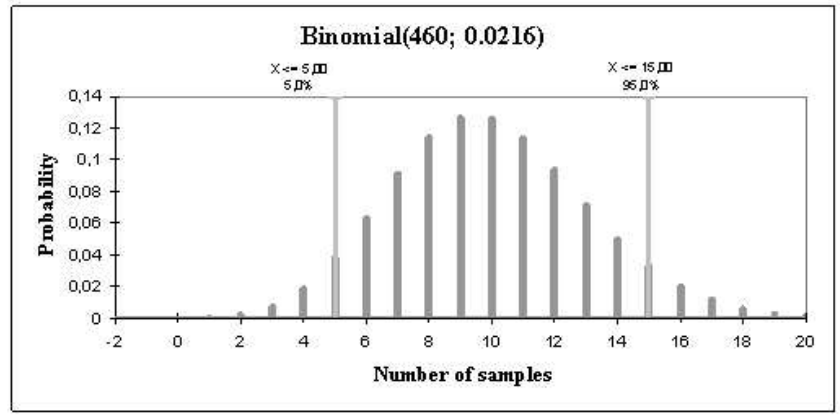

Figure 1b. Probability Distribution of presence-absence, 2 pesticide residue 2003-2007

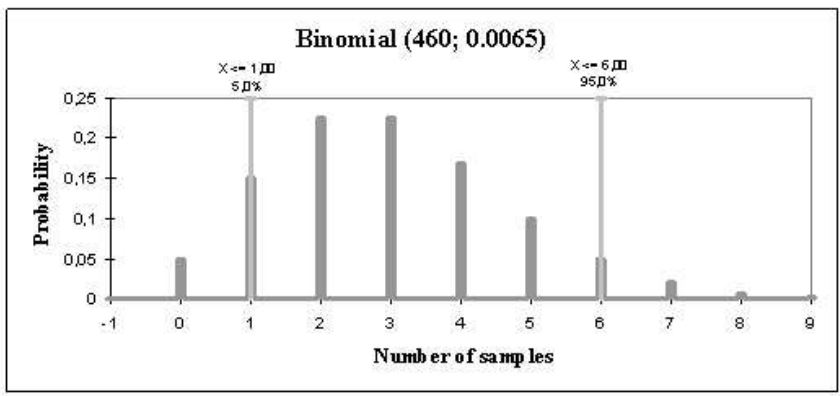

Figure 1c. Probability Distribution of presence-absence, 3 pesticide re sidue 2003-2007

$190 \times 254 \mathrm{~mm}(96 \times 96 \mathrm{DPI})$ 


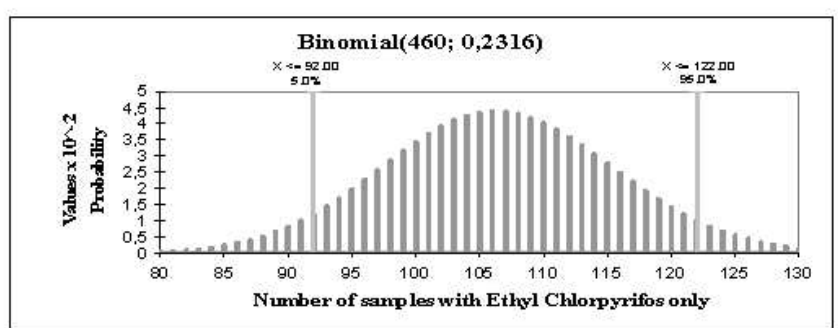

Figure 2a. Probability Distribution of presence-absence, Ethy Chl onpyrifos 2003-2007

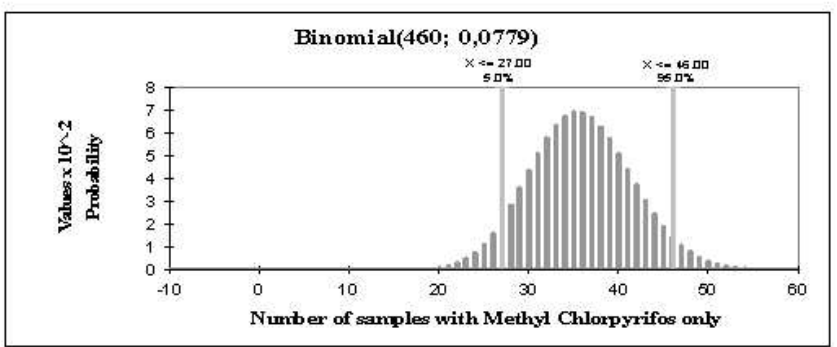

Figure 2b. Probability Distribution of presence-absence, Methyl Chlonpyrifos 2003-2007

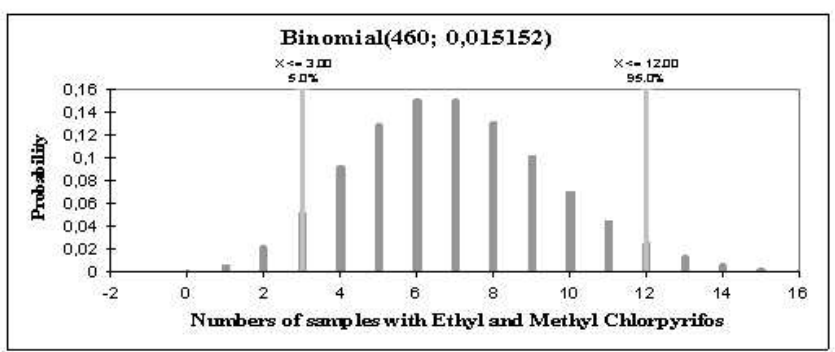

Figure 2c. Probability Distribution of presence-absence, Ethyl + Methyl Chl onpyrifos 2003-2007

$190 \times 254 \mathrm{~mm}(96 \times 96 \mathrm{DPI})$ 
Table 1. Sample Numbering

\begin{tabular}{|c|c|c|}
\hline Year & Percentage & Samples Number \\
\hline 2003 & 15.21 & $\mathbf{7 0}$ \\
\hline 2004 & 20 & $\mathbf{1 5 8}$ \\
\hline 2005 & 34.35 & $\mathbf{1 0 0}$ \\
\hline 2006 & 21.74 & $\mathbf{4 0}$ \\
\hline 2007 & 8.7 & $\mathbf{4 6 0}$ \\
\hline $2003-2007$ & 100 & \\
\hline
\end{tabular}


Table 2. Results of qualitative analysis

\begin{tabular}{|c|c|c|c|c|}
\hline & $\begin{array}{l}\text { Samples } \\
\text { Numbers }\end{array}$ & $\begin{array}{c}\text { Samples with } 1 \\
\text { residue }\end{array}$ & $\begin{array}{c}\text { Samples with } 2 \\
\text { residues }\end{array}$ & $\begin{array}{c}\text { Samples with } 3 \\
\text { residues }\end{array}$ \\
\hline 2003 & 70 & $\begin{array}{l}11(7-17) \\
p=0.1667\end{array}$ & $\begin{array}{c}1(0-4) \\
P=0.0278\end{array}$ & $\begin{array}{c}1(0-4) \\
p=0.0278\end{array}$ \\
\hline 2004 & 92 & $\begin{array}{l}31(24-39) \\
P=0.3404\end{array}$ & $\begin{array}{c}1(0-4) \\
P=0.0213\end{array}$ & 0 \\
\hline 2005 & 158 & $\begin{array}{l}69(59-79) \\
p=0.4375\end{array}$ & $\begin{array}{l}5 /(2-10) \\
P=0.0375\end{array}$ & 0 \\
\hline 2006 & 100 & $\begin{array}{l}18(12-25) \\
P=0.1863\end{array}$ & $\begin{array}{c}2(0-6) \\
p=0.0294\end{array}$ & $\begin{array}{c}1(0-4) \\
P=0.0196\end{array}$ \\
\hline 2007 & 40 & $\begin{array}{l}11(7-16) \\
P=0.2857\end{array}$ & 0 & 0 \\
\hline $2003-2007$ & 460 & $\begin{array}{c}140(124-157) \\
p=0.3052\end{array}$ & $\begin{array}{c}9(5-15) \\
p=0.0216\end{array}$ & $\begin{array}{c}2(1-6) \\
p=0.0065\end{array}$ \\
\hline
\end{tabular}

(M.L. $\min .5 \% ; \max 95 \%)$ 
Table 3. Total Quantitative Analysis (2003-2007)

\begin{tabular}{|c|c|c|c|c|c|c|c|c|c|}
\hline $\begin{array}{l}9 \\
10 \\
11 \\
12 \\
13 \\
14 \\
15 \\
16\end{array}$ & 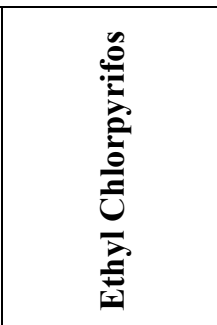 & 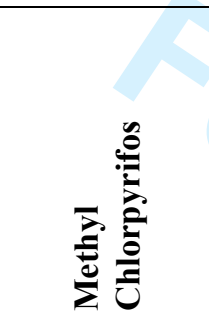 & $\frac{\overline{0}}{\frac{0}{0}}$ & 竞 & 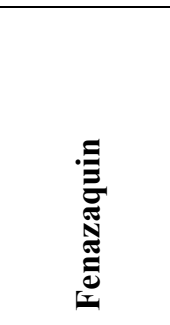 & 总 & 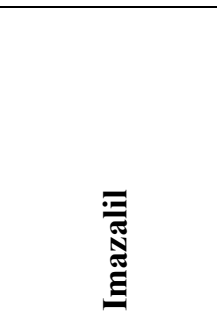 & 高 & 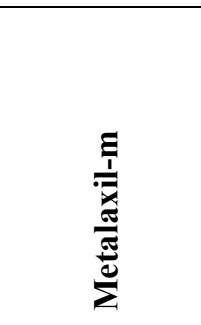 \\
\hline $\begin{array}{l}1 \nmid(\mathbf{R S D})_{\mathrm{R}} \\
1 \oint\end{array}$ & $8.1 \%$ & $8.0 \%$ & $18.3 \%$ & $5.2 \%$ & $15.2 \%$ & $6.2 \%)$ & $6.0 \%)$ & $12.5 \%)$ & $15.5 \%)$ \\
\hline $\begin{array}{l}19 \text { Positive samples } \\
20\end{array}$ & $106(23.04 \%)$ & $35(7.60 \%)$ & $3(0.65 \%)$ & $4(0.87 \%)$ & $2(0.43 \%)$ & $4(0.87 \%)$ & $5(1.08 \%)$ & $2(0.43 \%)$ & $3(0.65 \%)$ \\
\hline $\begin{array}{l}21 \text { MRL ( mg/Kg) } \\
22\end{array}$ & 0.3 & 0.5 & 2 & 1 & 0.2 & 2 & 5 & 2 & 0.5 \\
\hline $\begin{array}{l}2 \$ \text { Mean of Positive } \\
24 \\
2 \$ \text { Samples (mg/Kg) } \\
2 \phi\end{array}$ & $0.07 \pm 0.07$ & $0.56 \pm 0.57$ & $6.69 \pm 11.53$ & $0.003 \pm 0.001$ & $0.03 \pm 0.03$ & $0.04 \pm 0.05$ & $3.30 \pm 0.90$ & $0.02 \pm 0.01$ & $0.033 \pm 0.003$ \\
\hline 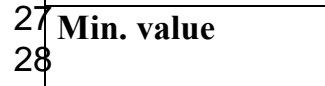 & 0.003 & 0.04 & 0.003 & 0.002 & 0.004 & 0.001 & 2.38 & 0.02 & 0.03 \\
\hline $\begin{array}{l}29 \text { Max value } \\
30\end{array}$ & 0.29 & 2.05 & 20 & 0.004 & 0.05 & 0.12 & 4.8 & 0.03 & 0.04 \\
\hline $\begin{array}{l}34 \text { Best } \\
32 \\
33 \text { Distribution } \\
34\end{array}$ & $\begin{aligned}= & \text { RiskNormal } \\
& (0.07 ; 0.07)\end{aligned}$ & $\begin{aligned}= & \text { RiskNormal } \\
& (0.56 ; 0.57)\end{aligned}$ & $\begin{array}{c}=\text { RiskTriang } \\
(0.003 ; 6.69 ; 20)\end{array}$ & * & * & * & $\begin{aligned}= & \text { RiskNormal } \\
& (3.30 ; 0.9)\end{aligned}$ & * & * \\
\hline
\end{tabular}

* Distribution absent for low number of samples 